\title{
Automated Two Phase to Three Phase Converter in Power Systems and Monitored By using IOT
}

\author{
D.Gayathri, R.Suganya, K.Soumiyaa
}

\begin{abstract}
Frequency is the main parameters of the power system. The frequency parameters are varied due to the several factors. One of the main factors of frequency variation is caused by the load mismatches In under frequency condition the losses are hugely increased at when the power consumed by the loads. In the over frequency condition, some of the load connection are broken and it creates the loss to the systems. The over frequency condition occurred due to the greater input power in the loads. In both the cases, the system frequency gradually decreases from the power system's obligatory frequency range. it causes tripping off of the substation and also ruining the entire system. The paper shows a magnificent method to employing a smart meter to overcome the destructions in the power systems
\end{abstract}

Index Terms-Mathematical Model, Three phase converter, IOT , Fuzzy logic control,PID controller

\section{INTRODUCTION}

Nowadays a several research and industrial applications are focuses to provide a simple and easy control algorithms and strategies. These algorithms are reduced the difficulty of the process and systems and enhance the motility of the controller. Most of the systems are not able to characterized by the conventional methods. Hence, these model-based design approaches may not provide satisfactory solutions.

Hence we implement the fuzzy logic controller in the the power system to improve the flexibility. it is based on fuzzy logic theory and employ a mode of approximate reasoning that resembles the decision making process of humans. Fuzzy logic control provides a gratification results of a control system.

Fuzzy logic is easily understandable by the human expert. It provides the conventional controllers with certain level of artificial intelligence to overcome the disadvantages of PID controllers . A FLC is used over a vast area to settle down the problems in unknown conditions and vague environments. In our project, Fuzzy Logic Controller (FLC) gives the adequate results. . By using the fuzzy logic controller to reduce the stability error through adding a pole to the origin of the system. In uncontrolled condition of the controller cause the peak overshoot problems, oscillations and negative overshoots. Fuzzy logic controller provides excellent result than the conventional PID controllers. The dynamics of the system is well monitored, resolved and controlled by the fuzzy logic controller.

\section{MATHEMATICAL MODELING OF LOAD}

Most of the load on the power system have a electrical drives. These drives are resistive in nature because it involves the lighting fields. The rotating devices are fundamentally a composite of the resistive and inductive components. The speed-load characteristic is given by

$\Delta \mathrm{Pe}=\Delta \mathrm{PL}+\mathrm{D} \Delta \omega$

Where $\Delta \mathrm{PL}$ load changes at non frequency state

$\mathrm{D} \Delta \omega$ is load changes at the condition of sensitive frequency changes

\section{A. Modeling And Controlling of Load Frequency}

Multiple loads are connected to the system, further frequency and speed are changed with the governor characteristics as the load changes. There is no need to maintain the frequency at a certain limit or constant in a system then the engineer is not necessary to alter the parameters of the generator. By adjusting the speed of the turbine to maintain the frequency at certain limit. The speed of the turbine is modify by the speed characteristics of generator. The modification of load can be done in two generating stations If the two generating stations running at parallel they causes difficulty in power systems. A tie line connects the two separated generated stations. There are three possibility of regulations are made in the power system to obtain the constant frequency. They are flat regulation, parallel regulation and flat tie line regulation. The flat regulation is applied at the load changes appeared at either a $\mathrm{A}$ or $\mathrm{B}$ and the generation of $\mathrm{A}$ is alone to regulate to get a constant frequency. Second one is parallel regulation, it regulates both $\mathrm{A}$ and $\mathrm{B}$ generations. Third one is if a system needs to change the frequency at particular area of the generator in such cases the flat tie line regulation is used.

\section{BLOCK OF THWO PHASE TO THREE PHASE CONVERTER}

Figure1 shows the simplified block diagram of automated two phase to three phase converter. The power is get from the power supply (transformer) and it is given to the Microcontroller . 


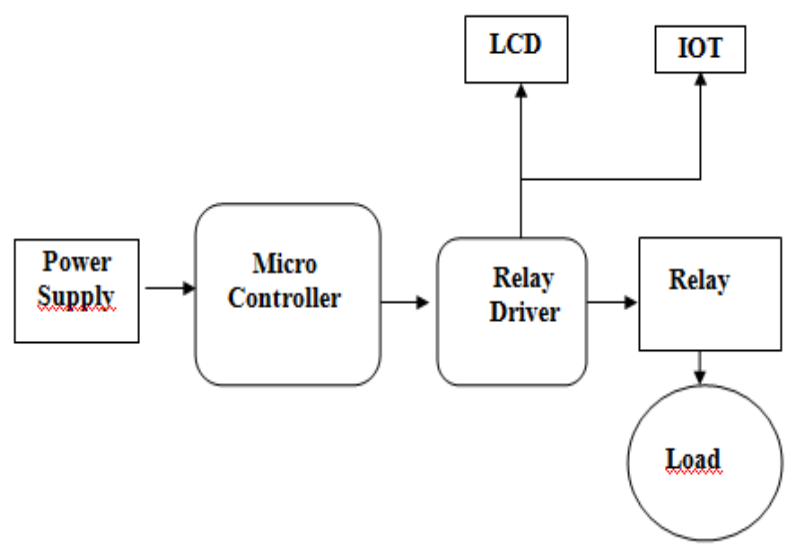

Figure 1 . Basic block diagram of two phase to three phase converter

Relays are employed to drive the load and the changed output is displayed in the LCD and also monitored by IOT server

\section{IV . HARDWARE REQUIREMENTS}

\section{A. 8051 Microcontroller}

8051 microcontroller is designed by Intel in 1981. It is an 8 -bit microcontroller. It is available in fourty pin dual in line package. The 8051 microcontroller is operated by using the $+5 \mathrm{~V}$ power supply and the pin number 20 is grounded. There are four types of ports are in 8051 microcontroller. All these four types are connected to get address and data from the devices. The reset option is also present in 8051 micro controller for preset the values.

\section{B. Power Supply}

Power supply is separated from the live circuit. A module should not be connected directly to the live circuit. Optical isolated technique is used to isolate the power supply. Isolated power supply is necessary to protect against the leakage currents. Because of its isolation the stray voltages are not occurred, which could cause a fluttered display

\section{C. $L C D$}

Liquid crystal displays (LCDs) are used to indicating the variations in frequencies for the visual representation. It composite the characters of both liquids and crystals. The LCD has low power consumption than LED. It displays characters in segmental and matrix forms.

\section{Relays}

A relay works on the principle of electromagnet. In most of the applications the relay act as a switches. The relays are used to provide the small and large current to the circuits. A lamp is used to provide a small current and the electric motor is employed to provide a large current to the circuit by using these relays.

\section{SOFTWARE REQUIREMENTS}

\section{A. Embedded $C$}

An embedded system is a embedded of three components in a circuit

It embeds both hardware and software .

$\mathrm{C}$ code is reliable and portable to written in the circuit.
This language is mostly used to program the controller because of its adaptability.

Embedded systems are controllers with on chip control.

\section{B. Keil C Compiler}

The $\mathrm{C}$ programming plays a vital role in performing functions by the processor. It is more reliable, portable and easily understandable by the users. . keil $\mathrm{C}$ complier are discovered to solve the complex problems occred in programming. The ground up implementation provides the fast and compact code for the 8051 microprocessor. The Cx51 is the advance version of the C Compiler to enchance complete implementation of $\mathrm{C}$ language. Cx51 increases affability and also increases the speed of the code

\section{SOFTWARE IMPLEMENTATION OF CONVERTER CIRCUIT}

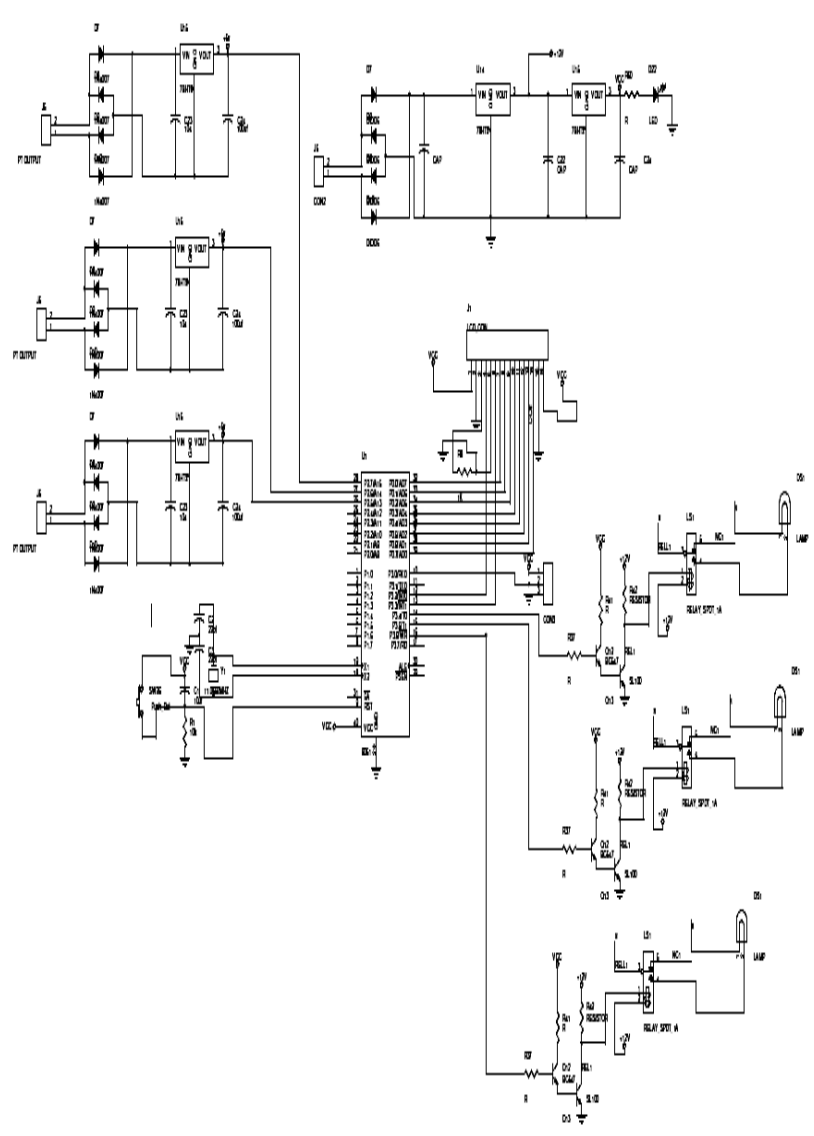

Figure 2. Software implementation of two phase to three phase converter

\section{HARDWARE IMPLEMENTATION OF CONVERTER CIRCUIT}

In this paper used three sets of step down transformer, respective voltage regulator ,microcontroller, and Bluetooth chip. Three phase supply is given to the power system ,the supply ranges in $12 \mathrm{~V}, 1 \mathrm{~A}$. Initially the setup operates in three phase, when suddenly one phase kept cutoff due to some sudden conditions, the operation cannot be discontinued

Published By: 


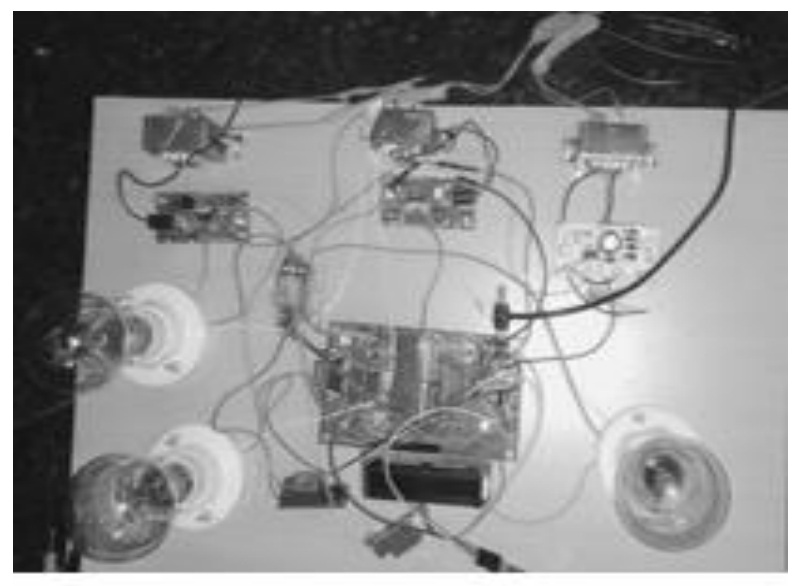

Figure 3 . Hardware implementation of two phase to three phase converter

The two phase condition provides the shutdown or off condition of motor. Hence the two phase is converted to three phase by obtaining power from the transformer .

The changed values can be read and displayed by using LCD and can be stored in mobile by using IOT

\section{SAMPLE PROGRAMS OF MICROCONTROLLER \& RESULTS}

\#include <REGX52.H>

\#defineSERIAL_NEXT_LINE

serial_trans(0x0d); serial_trans(0x0a);

\#define ENTER serial_trans(0x0d);

typedef unsigned char U1;

sbit $\mathrm{RS}=0 \mathrm{xA} 6$;

sbit $\mathrm{EN}=0 \times \mathrm{xA} 7$;

sbit $r=0 \times 90$;

sbit $\mathrm{y}=0 \times 91$;

sbit $\mathrm{b}=0 \times \mathrm{x} 92$;

sbit tank1 = 0x93;

sbit tank2 = 0x94;

sbit relyV = 0xb4;

sbit relyR $=0 x b 5$;

sbit relyY $=0 x b 6$;

sbit relyB $=0 \mathrm{xb} 7$

sfr lcd_dat $=0 \times 80$;

\}

void sms_read(void)

\{

if $(r==0 \& \& y==0 \quad \& \& b==0)$

\{

relyR=1;relyY=1;relyB=1;

lcd_line("ALL PHASE ACTIVE",0x80);

lcd_line(" MOTOR ON ",0xC0);

sms_send ("ALL PHASE ACTIVE, MOTOR

ON","9865862045");

lcd_line("R,B PHASE ACTIVE",0x80);

lcd_line(" MOTOR OFF ",0xC0);

\}

else if $(r==0 \& \& y==0 \& \& b==1)$

\{

relyR=0;relyY=1;relyB=0;

lcd_line("R,Y PHASE ACTIVE",0x80);

lcd_line(" MOTOR OFF ",0xC0);
sms_send("R,Y PHASE ACTIVE, MOTOR

OFF","9865862045");

\}

else if $(\mathrm{r}==1 \quad \& \& \mathrm{y}==1 \quad \& \& \mathrm{~b}==0)$

\{

relyR=0;relyY=0;relyB=0;

lcd_line("B PHASE ACTIVE ",0x80);

lcd_line(" MOTOR OFF ",0xC0);

sms_send("B PHASE ACTIVE, MOTOR

OFF","9865862045");

\}

else if $(r==1 \quad \& \& \quad y==0 \quad \& \& b==1)$

\{

relyR=0;relyY=0;relyB=0;

lcd_line("Y PHASE ACTIVE ",0x80);

lcd_line(" MOTOR OFF ",0xC0);

\}

else if $(r==0 \quad \& \& \quad y==1 \quad \& \& b==1)$

\{

relyR=0;relyY=0;relyB=0;

lcd_line("R PHASE ACTIVE ",0x80);

lcd_line(" MOTOR OFF ",0xC0);

sms_send("R PHASE ACTIVE, MOTOR

OFF","9865862045");

\}

else if $(r==1 \& \& y==1 \quad \& \& b==1)$

\{

relyR=0;relyY=0;relyB=0;

lcd_line("NO PHASE ACTIVE ",0x80);

lcd_line("POWER SHUTDOWN ",0xC0);

sms_send("NO PHASE ACTIVE, MOTOR

OFF","9865862045");

\})

else if $(r==1 \quad \& \& y==0 \quad \& \& b==0)$

\{

relyR=0;relyY=0;relyB=0;

lcd_line("Y,B PHASE ACTIVE",0x80);

lcd_line(" MOTOR OFF ",0xC0);

sms_send("Y,B PHASE ACTIVE, MOTOR

OFF","9865862045");

\}

else if $(r==0 \quad \& \& y==1 \quad \& \& b==0)$

\{

relyR=0;relyY=0;relyB=0;

void sms_delete(void);

void gsm_module_init(void);

void sms_send1(unsigned char *message);

unsigned int $\mathrm{m}=5, \mathrm{n}=5 ; \mathrm{k}$;

unsigned char $\mathrm{j}$;

unsigned char rec_dat[99];

void main()

\{// $\mathrm{EA}=1$;

// ET0=1;

TMOD=0X22;

$\mathrm{TH} 0=0 \mathrm{XCC}$;

// TR0 $=1$;

$\mathrm{P} 3=0 \mathrm{X} 0 \mathrm{~F}$;

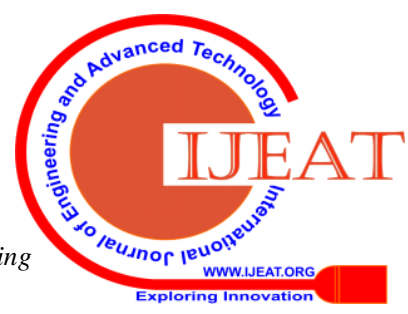




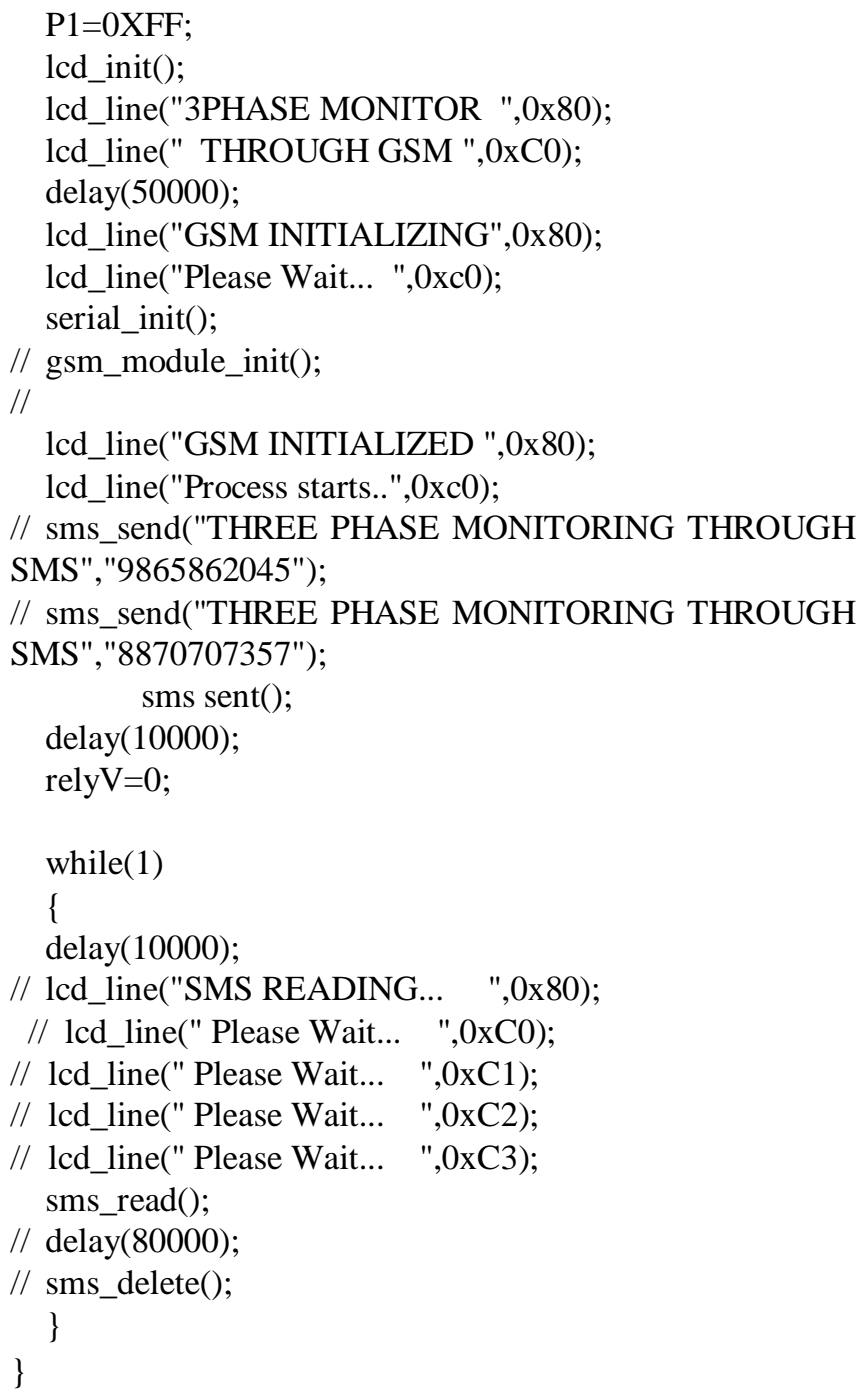

\section{CONCLUSION}

In this project, we can avoid discontinuity of power system operation.

Here we are using fuzzy logic control technique in order to analyze and characterize the system in the time of disconnection of load.

The overall values which is obtained from the conversion of two phase to three phase would be stored in separate server with a separate file using internet of things.

This provides easy monitoring, data collection and detection of location.

\section{REFERENCES}

1. Shivanagouda.B.Patil, M. S. Aspalli," Operating Three Phase Induction Motor Connected to Single Phase Supply ," International Journal of Emerging Technology and Advanced Engineering, ISSN 2250-2459, Volume 2, Issue 11, November 2012.

2. Cichocki and R. Unbehaven, Neural Networksfor Optimization and Signal Processing, 1st ed. Chichester,U.K.: Wiley, 1993, ch. 2, pp. 45-47.

3. Dong choon Lee, member IEEE and YoungSin Kim," Control of Single-Phase-to-Three-Phase AC/DC/AC PWM converter for Induction Motor Drives," IEEE TRANSACTIONS ON INDUSTRIAL ELECTRONICS, VOL. 54, NO. 2, APRIL 2007

4. M.Anandhavelan, M. Aravind raj, "Monitoring and Control of Three Phase Induction Motor Using Iota Based
Concept" IOSR Journal of Engineering (IOSRJEN) www.iosrjen.org ISSN (e): 2250-3021, ISSN (p): 2278-8719 PP 27-31

5. Anbuhganapathy, N. Sivanandham "Control of Three Phase Induction Motor by Ardiuno with IoT" IJSRD International Journal for Scientific Research \& Development| Vol. 6, Issue 03, 2018 | ISSN (online): 2321-0613

6. Devanand K. Patil*1, Kaveri G. Avhad2, Prathamesh C Johore3 "A REVIEW PAPER ON CONTROLLING OF THREE PHASE \& SINGLE PHASE ELECTRICAL EQUIPMENT BY USING IOT, GSM \& WI-FI" ISSN2350-0530(O), ISSN- 2394-3629(P) ICV (Index Copernicus Value) 2015: 71.21 IF: 4.321 (CosmosImpactFactor), 2.532 (I2OR) InfoBase Index IBI Factor 3.86

7. Hareharan V 1 , Sasikumar M2 ,ChandraKala P3 "EFFICENCY MONITORING OF THE THREE PHASE INDUCTION MOTOR USING IoT "Vol-4 Issue-2 2018 IJARIIE-ISSN(O)-2395-4396

8. V.S.D Rekha "Induction Motor Condition Monitoring and Controlling Based on IoT" International Journal of Electronics, Electrical and Computational System IJEECS ISSN 2348-117X Volume 6, Issue 9 September 2017

9. Seenivasan V1,Ponkumar K1 ,Venkatraman R1, "INDUCTION MOTOR CONDITION MONITORING AND CONTROLLING BASED ON IOT" International Research Journal of Engineering and Technology (IRJET) e-ISSN: 2395-0056 Volume: 06 Issue: 3 | Mar 2019 www.irjet.net p-ISSN: 2395-007

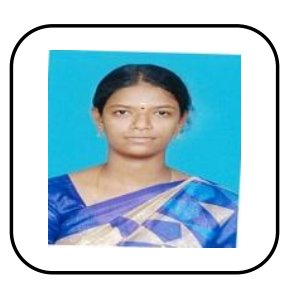

Gayathri was born on May 24, 1994. She received the BE. from Anna University, in 2011 and ME from Annamalai University in 2015, respectively.In 2017, she joined IFET College of Enginnering , in Tamil Nadu, as a Professor. His representative published articles lists as follow: Zigbee based home appliance control using voice recognition for disabled people (IJR,2018) Comparative study of MRAC and STC controllers for a Nonlinear interacting coupled Tank Process(IJPAM,2018) Controlling the Cooling operation of Transformer using Embedded PLC (IJRR 2019) "Multiloop adaptive controllers for a nonlinear interacting coupled tank process", International Journal of Engineering Research and Technology (IJERT,2017) Designing Cascaded Current Fed Push Pull Converter and Spwm Inverter for Residential Application (IOSR,2019)

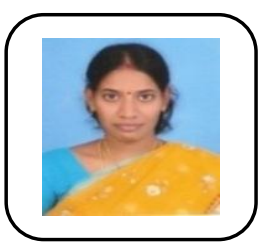

R.Suganya has rich experience of 7 years as an academician. At present she is an Senior Associate Professor of Electrical and Electronics Engineering at IFET College of Engineering, Villupuram, India She has done M.E in the year 2008 from Thiagarajar College of Engineering, Madurai. Her areas of interest are Power system Structuring, Voltage stability, Electricity Deregulation etc 